\title{
Analytical Model for Distortion Prediction in Wire + Arc Additive Manufacturing
}

\author{
Jan Roman Hönnige ${ }^{a,{ }^{*}}$, Paul Colegrove ${ }^{b}$ and Stewart Williams ${ }^{c}$ \\ Welding Engineering and Laser Processing Centre (WELPC), Cranfield University, Cranfield, \\ Bedfordshire, MK43 OAL, UK \\ aj.honnige@cranfield.ac.uk, bp.colegrove@cranfield.ac.uk, cs.williams@cranfield.ac.uk
}

\begin{abstract}
Keywords: Residual Stress, Bending Distortion, Neutron Diffraction, Geometry Factor, Material and Process Factor, Critical Wall Height
\end{abstract}

\begin{abstract}
An analytical model was developed to predict bending distortion of the base-plate caused by residual stresses in additively manufactured metal deposits. This avoids timeconsuming numerical simulations for a fast estimation of the expected distortion. Distortion is the product of the geometry factor $\boldsymbol{K}$, which is determined by the cross-section of substrate and deposit, and the material and process factor $\boldsymbol{S}$, which is the quotient of residual stress and the Young's Modulus. A critical wall height can be calculated for which the structure distorts the most. This critical height is typically less than 2.5 times the thickness of the substrate. Higher walls increase the stiffness of the cross-section and reduce the distortion with increasing height.
\end{abstract}

\section{Introduction}

Wire + Arc Additive Manufacturing (WAAM) can be used to manufacture large-scale near-netshape preforms for structural components with medium complexity [1]. Typical materials are structural metals, such as steel, titanium, Inconel or aluminium alloys. Residual Stresses are amongst the largest challenges in WAAM. They can cause serious distortion [2] and premature failure during the deposition or in service of the component [3]. It was widely reported using different experimental approaches and numerical simulations that stresses can reach values between $50 \%$ and $100 \%$ of the materials yield strength [2-7]. Thermal stress relieving can be used to eliminate these stresses [8]. However, unclamping the part after the deposition causes distortion before the part can be heat treated. The magnitude of the distortion depends on the residual stresses and therefore on the thermo-mechanical history of the deposition, as well as the material characteristics and the geometry. The repetitive deposition procedure requires extensive computing in numerical approaches to capture the full thermal history, required for stress calculation [6]. Simplified models can be accurate and more efficient [9], but parametric investigations would still require the simulation of each individual case. This makes it time consuming to compare cross-sectional variations. Analytical approaches, as they exist for buttwelding [10] or cladding processes [11], can estimate distortion fast and effortless. Colegrove et al. [2] proposed the analogy of the behaviour of residual stresses in WAAM and bending distortion according to cantilever beam theory, which will be elaborated and validated in this paper.

\section{Methodology}

Analytical Model for Distortion Prediction. Previous investigations allowed fundamental assumptions for this analytical approach. Uniform residual stresses are produced along the wall height during deposition, as shown in the schematic in Figure 1 (a). The balancing compressive stresses in the substrate are assumed constant as well with a non-continuous transition in the interface. Unclamping causes distortion and redistribution of the stresses in a way that these 
tensile stresses drop linearly towards the top of the wall (Figure 1 (b)) [2]-[7]. This stress development and redistribution is furthermore assumed to be constant along the length $L$ of the wall, disregarding any edge effects. The distortion $w(x)$ is therefore a function of the length $L$ and the constant curvature $\kappa$ (the inverse of the curvature radius $\rho$ ), as illustrated in Figure 1 (c). The analytical model assumes a deposition of a straight linear wall. Wall width $W W$ and wall height $W H$, as well as substrate thickness $t$ and width $b$ are geometrical variables. Figure 1 (d) and Eq. (1) and (2) show how these variables can be used to find the centroid of the substrate and deposit. The centroid of the overall area $z_{0}$ is also the location of the neutral axis of the T-section and can be calculated using Eq. (3) to determine the moment of inertia $I_{y y}$ with Eq. (4).

$$
\begin{aligned}
& z_{s}=\frac{1}{2} t \\
& z_{d}=\frac{1}{2} W H+t \\
& z_{0}=\frac{\sum z_{s, i} A_{i}}{\sum A_{i}}=\frac{\left(z_{d} \times W W \times W H\right)+\left(z_{s} \times b \times t\right)}{W W \times W H+b t} \\
& I_{y y}=\frac{1}{12}\left(b t^{3}+W W \times W H^{3}\right)+b t \times\left(z_{0}-z_{s}\right)^{2}+W W \times W H \times\left(z_{d}-z_{0}\right)^{2} \\
& F_{d}=F_{s}=F=-\iint \sigma_{x x, d}(y, z) * d A(W W, W H) \\
& F=\sigma_{x x} * W W * W H \\
& M=F_{d}\left(z_{d}-z_{0}\right)+F_{s}\left(z_{0}-z_{s}\right)=F\left(z_{d}-z_{s}\right)=F\left(\frac{1}{2} t+\frac{1}{2} W H\right) \\
& \text { material \& process factor } S \text { geometry factor } K \\
& \kappa\left[\frac{1}{m}\right]=\frac{1}{\rho}=\frac{M}{E I_{y y}}=\quad \frac{\widetilde{\sigma_{x x}}}{E} \quad \times \frac{\widetilde{W W \times W H \times(t+W H)}}{2 I_{y y}}=\mathrm{S}\left[\frac{M P a}{G P a}\right] \times K\left[\frac{1}{m}\right]
\end{aligned}
$$

(a)

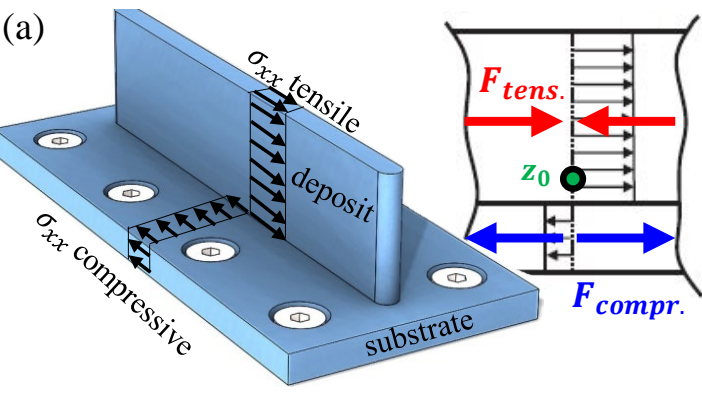

(c)

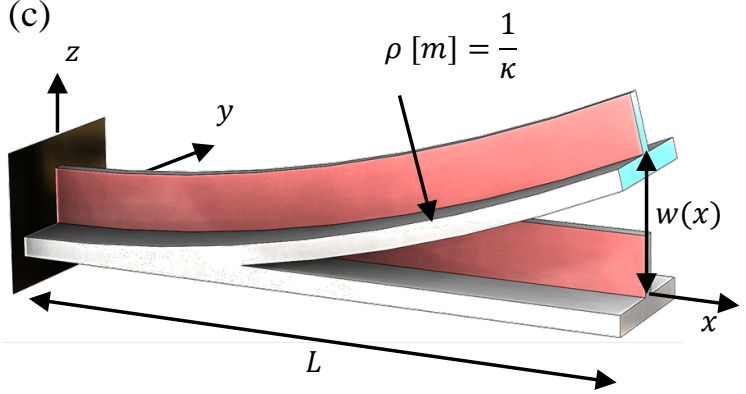

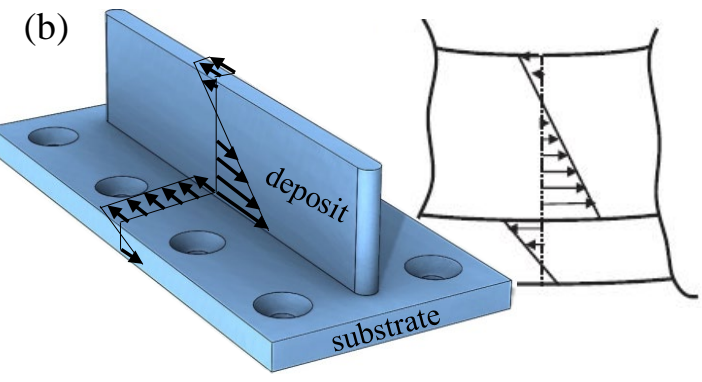

(d)

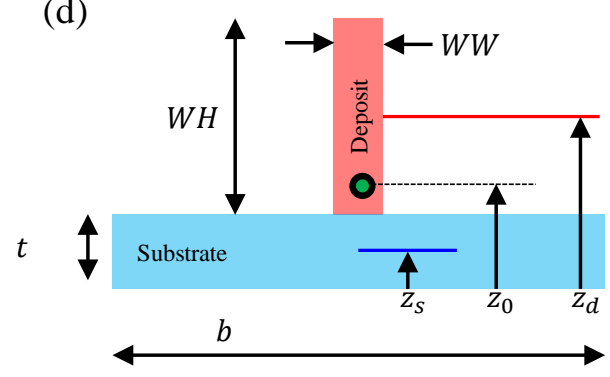

Figure 1: (a) unbalanced stress field development during the WAAM deposition; (b) redistributed and self-balanced stress field after distortion; (c) cantilever beam with constant curvature $\rho$ and cross-section along the length $L$ and the distortion $w(x)$; (d) variables in crosssection. 
The internal forces $F_{d}$ caused by the residual stresses $\sigma_{x x}$ in the deposit can be calculated with Eq. (5) and act at the centre gravity of this integrated volume. The compressive stress in the substrate $F_{s}$ must be equal to $F_{d}$. Since the stresses were assumed to be constant across the rectangular cross-section, the internal force $F_{d}$ acts at $z_{d}$ and $F_{s}$ acts at $z_{s}$ and the magnitude simplifies as per Eq. (6). The location of the concentrated forces $F_{d}$ and $F_{s}$ result in a bending moment around the neutral axis, which is calculated with Eq. (7).

The curvature $\kappa$ can now be calculated using Eq. (8), in which the quotient of the Young's modulus and the residual stress define the process and material factor $S$, while the geometry factor $K$ is entirely dependent on the four cross-sectional variables. The value of the tensile residual stress $\sigma_{x x}$ needs to be available for distortion prediction and depends on material characteristics and the thermal history. It can be determined experimentally [2]-[7] or analytically [11].

Stress redistribution. The redistributed residual stress field after distortion (Figure 1 (b)) is the sum of the initial stress field before unclamping (Figure 1 (a)) and the stress caused by the internal bending moment, according to Eq. (9), where $\mathrm{z}=0$ is located in the neutral axis $z_{0}$. Both, the bending moment $M$ and the $I_{y y}$ are constant along the length. The equilibrium residual stress field is therefore the as-clamped stress field, after pivoting around the neutral axis. The stress gradient, which drops linearly towards the top of the wall can be calculated using Eq. (10).

$$
\begin{aligned}
& \sigma_{x x, r e s}(z)=\sigma_{x x}+\frac{M}{I_{y y}} \times z \\
& \frac{\Delta \sigma_{\mathrm{xx}}}{\Delta \mathrm{z}}=\frac{M}{I_{y y}}
\end{aligned}
$$

Validation of Prediction for Distortion and Stress Redistribution. To validate the distortion prediction three geometrically identical walls were built with WAAM using Ti-6Al-4V, In718 and the aluminium alloy AA2319. The dimensions were $L=250, W W=6, W H=22, t=7$ and $b=60$ (Figure 1; units in $\mathrm{mm}$ ). Neutron diffraction strain measurements were performed for stress calculation using the ENGIN-X instrument at ISIS in Didcot for the Ti-6Al-4V [4] and aluminium [7] deposits and the SALSA beam line at ILL in Grenoble for the Inconel deposit ${ }^{1}$. For the latter an incoming wavelength of $1.62 \AA$ was used to diffract the $\{311\}$ crystallographic plane with an angle of $2 \theta=96.2^{\circ}$. The scan was performed along the vertical centreline in the middle of the deposit. All three specimen were scanned before and after unclamping. The constant longitudinal stress value in the clamped condition was used to predict the distortion and the associated stress gradients after redistribution to compare them with the experimental results.

\section{Results}

The geometry factor $K$ of any profile can be plotted numerically, as shown in Figure 2 (a). It is here plotted as a dimensionless function of practical ratios of $W H / t$ and $b / W W$, allowing the determination for any arbitrary combination of realistic wall geometries on any substrate. The cross-section in the present experiments has a value $K=68.57[1 / \mathrm{m}]$ (X in Figure 2 (a)). The critical wall height is defined as the value which gives the maximum value of $\mathrm{t}^{*} \mathrm{~K}$ in Figure 2 (a)). This is plotted as a function of $b / W W$ in Figure 2 (b). Walls that exceed the critical height result in increased stiffness of the cross-section and therefore reduce the bending distortion.

The determined residual stress from the neutron diffraction experiment are shown in Figure 3, from which the constant residual stress values for each alloy can be taken. The dashed lines are not trend lines, but the predicted stress redistribution using the analytical model. For this

\footnotetext{
${ }^{1}$ The instrument and typical methodology are described in Pirling et al. [12]
} 
geometry, the neutral axis $z_{0}$ almost coincides with the interface $(z=6.97 \mathrm{~mm})$, which is why the both stress plots, before and after unclamping meet in the interface.

The calculated results of the analytical model in Table 1 are compared with the measured stress gradients and the actual distortion of the specimen. The distortion of $200 \mathrm{~mm}$ long specimen was calculated using the constant curvature.

(a)

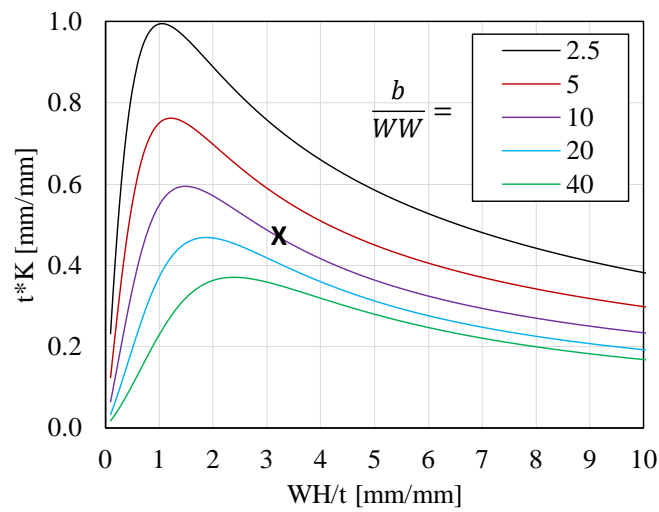

(b)

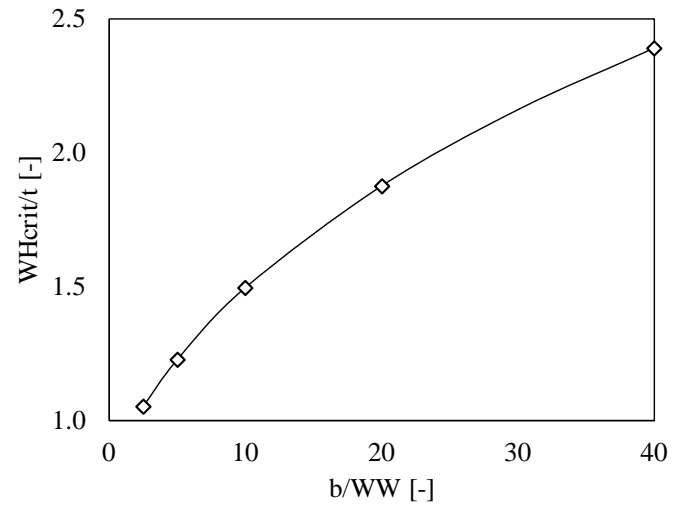

Figure 2: (a) Plot of the geometry factor $K$ for all dimensional ratios and (b) the critical wall height

(a) $\mathrm{Ti}-6 \mathrm{Al}-4 \mathrm{~V}$

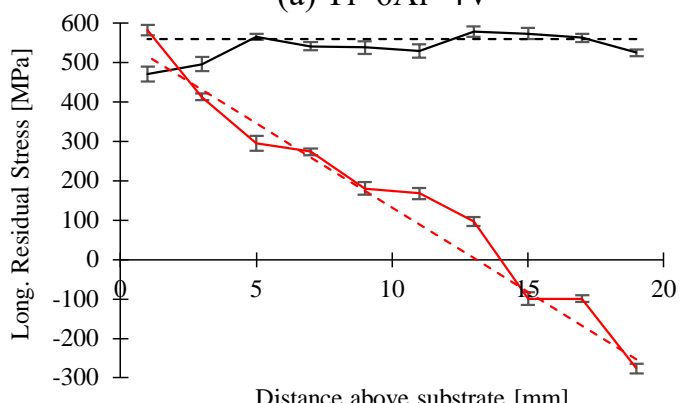

(c) $\operatorname{In} 718$

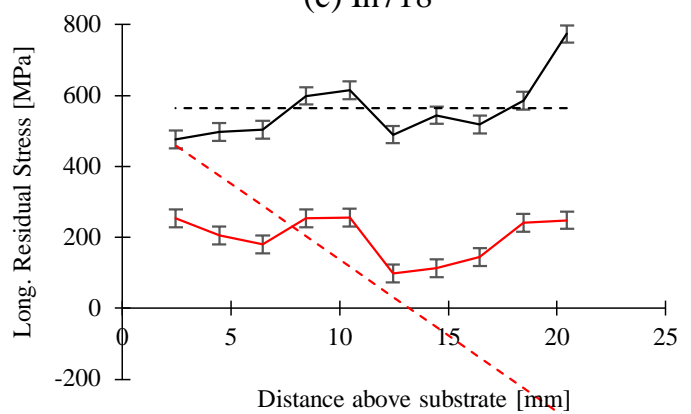

(b) AA 2319

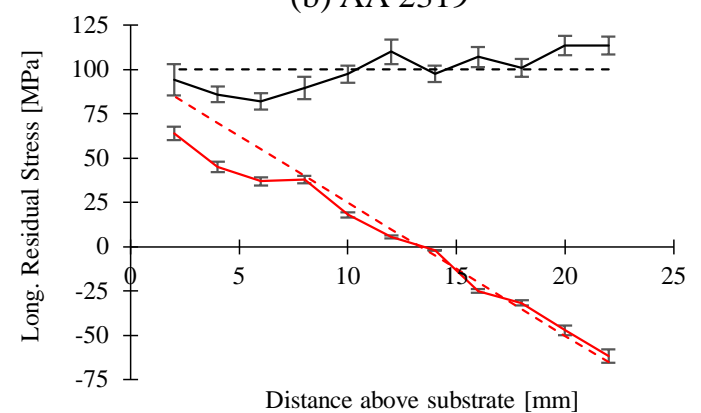

Distance above substrate $[\mathrm{mm}]$

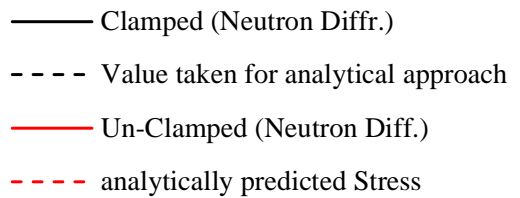

Figure 3: Measured Longitudinal Residual Stress using Neutron Diffraction and that predicted from the analytical model using identical wall geometries of (a) Ti-6Al-4V [4] (b) Aluminium AA 2319 [7] and (c) Inconel In718.

Table 1: Experimentally and analytically determined distortion characteristics for $K=68.57$ $[1 / m]$

\begin{tabular}{lllllrlrl}
\hline & $\begin{array}{l}\sigma_{\mathrm{xx}}[\mathrm{MPa}] \\
\text { exper. }\end{array}$ & $\begin{array}{l}\mathrm{S}[\mathrm{MPa} / \mathrm{GPa}] \\
\text { exper. }\end{array}$ & $\begin{array}{l}\kappa[1 / \mathrm{m}] \\
\text { analyt. }\end{array}$ & $\begin{array}{l}\rho[\mathrm{m}] \\
\text { analyt. }\end{array}$ & \multicolumn{2}{c}{$\mathrm{w}(\mathrm{L}=200 \mathrm{~mm})$} & \multicolumn{2}{c}{$\Delta \sigma / \Delta \mathrm{z}[\mathrm{MPa} / \mathrm{mm}]$} \\
analyt. & exper. & analyt. & exper. \\
\hline Ti-6Al-4V & 565 & 5 & 0.348 & 2.873 & 6.92 & 7.1 & 42.8 & 40 \\
In718 & 560 & 2.8 & 0.199 & 5.02 & 3.98 & 5.64 & 41.6 & 11 \\
AA 2319 & 130 & 1.75 & 0.092 & 10.84 & 1.85 & 1.75 & 7.58 & 7 \\
\hline
\end{tabular}




\section{Discussion}

The analytical model is in good agreement with the experimental results of titanium and aluminium deposition (Table 1). The Inconel results do not agree, which may be due to experimental errors, such as insufficient clamping and slip of the substrate during the deposition. As a result bending distortion may have occurred during the deposition already, leading to more distortion than predicted and less redistribution during unclamping. However, the good agreement with the other two alloys gives confidence that the analytical model can be used to provide a good first estimation of distortion and stress redistribution. A high geometry factor $\boldsymbol{K}$ and a high material and process factor $\boldsymbol{S}$ produce large distortion. Titanium alloys are therefore particularly susceptible due to high residual stresses ( 600 MPa) combined with a relatively low elastic modulus (113 GPa) [4]. Inconel and Steel are less susceptible due to their stiffness above $200 \mathrm{GPa}$ [2], while having comparable stresses. Aluminium is the least susceptible material due to very low residual stresses ( 100 MPa ) that over-compensate the low stiffness (72 GPa) [7]. The analytical model requires the input of these values. If no residual stress data is available, then assuming yield strength as residual stress is justifiable and represents the worst case scenario. The value can otherwise be estimated analytically based on thermal material properties [11].

Another interesting observation is the existence of a critical wall height $W_{\text {crit }}$ (Figure 2 (b)), which depends only on geometry and indicates the wall height that results in the greatest distortion. This value appears to be smaller than 2.5 times the thickness of the substrate for realistic geometries. Exceeding this wall height would result in reduced distortion. The figurative reason is that the added height increases the stiffness of the cross-section more than the bending moment increases. This results in shortening of the substrate, rather than in bending distortion. For example, building a $70 \mathrm{~mm}$ high wall (instead of $22 \mathrm{~mm}$ ) would reduce the geometry factor to $K=32.86$ and the final distortion of the titanium wall from $6.92 \mathrm{~mm}$ to $3.26 \mathrm{~mm}$. It should be noted that the critical wall height is independent of material and process.

For the purpose of comparing this approach with experimental results, simple geometries like a rectangular cross-section are easier to display, allowing the dimensionless and universally valid plot in Figure 2. However, more complex deposits can be predicted as well (e.g. several walls parallel to each other, non-rectangular cross-sections, cross-sectional variations along length, asymmetric double sided deposits, 2D deposits, stress gradients).

For more complex geometries, the integral in eq. (5) would have to be solved, assuming that the force in the deposit acts in the center of the integrated volume and the moment of inertia of the new cross-section has to be calculated accordingly.

\section{Conclusion}

The analytical model is a very effective tool for estimating the distortion and stress redistribution in additively manufactured structures on a substrate. The accuracy is reasonably good, considering the assumptions and boundary conditions. The main input is the as-deposited residual stress, which can be found experimentally. This value can otherwise be estimated analytically [11] or simply be assumed to be as high as the materials yield strength.

It was furthermore found that for any possible geometry combination there is a critical wall height, which would result in the greatest possible distortion for a particular AM process and material. This critical wall height is typically smaller than 2.5 times the thickness of the substrate. It is therefore also possible to determine the most-critical substrate geometry for a deposit in terms of bending distortion.

\section{Acknowledgement}

The findings were generated with the financial support of the WAAMMat program. 


\section{References}

[1] S. W. Williams, F. Martina, a. C. Addison, J. Ding, G. Pardal, and P. Colegrove, "Wire+Arc Additive Manufacturing,” Mater. Sci. Technol., vol. 32, no. 7, pp. 641-647, 2015.

[2] P. A. Colegrove, H. E. Coules, J. Fairman, F. Martina, T. Kashoob, H. Mamash, and L. D. Cozzolino, "Microstructure and residual stress improvement in wire and arc additively manufactured parts through high-pressure rolling,” J. Mater. Process. Technol., vol. 213, pp. 1782-1791, 2013.

[3] J. Zhang, X. Wang, S. Paddea, and X. Zhang, "Fatigue crack propagation behaviour in wire + arc additive manufactured Ti-6Al-4V: Effects of microstructure and residual stress," Mater. Des. Des., vol. 90, pp. 551-561, 2016.

[4] J. R. Hönnige, S. Williams, M. J. Roy, P. Colegrove, and S. Ganguly, "Residual Stress Characterization and Control in the Additive Manufacture of Large Scale Metal Structures," in 10th International Conference on Residual Stresses, 2016.

[5] F. Martina, M. J. Roy, B. A. Szost, S. Terzi, P. A. Colegrove, S. W. Williams, P. J. Withers, J. Meyer, and M. Hofmann, "Residual stress of as-deposited and rolled wire+arc additive manufacturing Ti-6Al-4V components,” Mater. Sci. Technol., vol. 0836, no. April, 2016.

[6] J. Ding, P. Colegrove, J. Mehnen, S. Ganguly, P. M. S. Almeida, F. Wang, and S. Williams, "Thermo-mechanical analysis of Wire and Arc Additive Layer Manufacturing process on large multi-layer parts,” Comput. Mater. Sci., vol. 50, no. 12, pp. 3315-3322, 2011.

[7] J. R. Hönnige, P. A. Colegrove, S. Ganguly, E. Eimer, S. Kabra, and S. W. Williams, "Control of Residual Stress and Distortion in Aluminium Wire + Arc Additive Manufacture with Rolling,” Addit. Manuf., vol. 22, pp. 775-783, 2018.

[8] J. R. Hönnige, P. A. Colegrove, B. Ahmad, M. E. Fitzpatrick, S. Ganguly, T. L. Lee, and S. W. Williams, "Residual stress and texture control in Ti-6Al-4V wire+arc additively manufactured intersections by stress relief and rolling,” Mater. Des., vol. 150, pp. 193-205, 2018.

[9] J. Ding, P. Colegrove, J. Mehnen, S. Williams, F. Wang, and P. S. Almeida, “A computationally efficient finite element model of wire and arc additive manufacture,” Int. J. Adv. Manuf. Technol., vol. 70, pp. 227-236, 2014.

[10]J. Winczek, R. Parkitny, K. Makles, and M. Kukuryk, “Analytical model of stress field in submerged arc welding butt joint with thorough penetration," in MATEC Web of Conferences MMS 2017, 2018, vol. 157, pp. 1-11.

[11]N. Tamanna, R. Crouch, I. R. Kabir, and S. Naher, "An analytical model to predict and minimize the residual stress of laser cladding process,” Appl. Phys. A Mater. Sci. Process., vol. 124, no. 2, pp. 1-5, 2018.

[12]T. Pirling, G. Bruno, and P. J. Withers, "SALSA-A new instrument for strain imaging in engineering materials and components,” Mater. Sci. Eng. A, vol. 437, pp. 139-144, 2006. 